\title{
FLOW TRAINING DIKES FOR THE WOURI ESTUARY IN DOUALA DC PROSPECTION OF SAND IN SHALLOW SEA WATER
}

\author{
Samuel N'Seke \\ Bruno Bagnérès \\ Richard Lagabrielle \\ Jean-Luc Mattiuzzo
}

\author{
CNPA - Director of Access - Douala - Cameroon \\ SOGREAH - Project Manager - Grenoble - France \\ LCPC - Chief of Soil Mechanics Division - Nantes - France \\ SEGG - General Manager - Chambery - France
}

The Port of Douala, which is located high up the Wouri River Estuary, is reached by means of a $50 \mathrm{~km}$ long access channel. The $25 \mathrm{~km}$ upper part of this channel currently requires intense and costly maintenance dredging. 20 years of dredging experience gained through three previous programs has led the Cameroon National Ports Authority (CNPA) to consider that an alternative solution with higher investment costs (flow training dikes) and reduced maintenance (by more than $60 \%$ ) is the most appropriate in terms of present benefits.

On behalf of the CNPA, French consultant Sogreah recently completed the final design and tender documents for the construction of $18 \mathrm{~km}$ of training dikes, tested previously on a largescale physical and mathematical model.

The following article describes an innovative geophysical method used for sand and gravel prospection in three selected areas of the estuary. This investigation, ordered by the CNPA, were performed by SEGG, a french firm specialised in geophysics, in collaboration with the LCPC, French public laboratory in the field of public works. The paper describes the principles of the method as developped by the LCPC and its application on the Wouri River site where SEGG has achieved a $184 \mathrm{~km}$ profiling survey during summer 1994 in order to estimate the volume of sand and gravel available in Wouri estuary for flow training dikes construction.

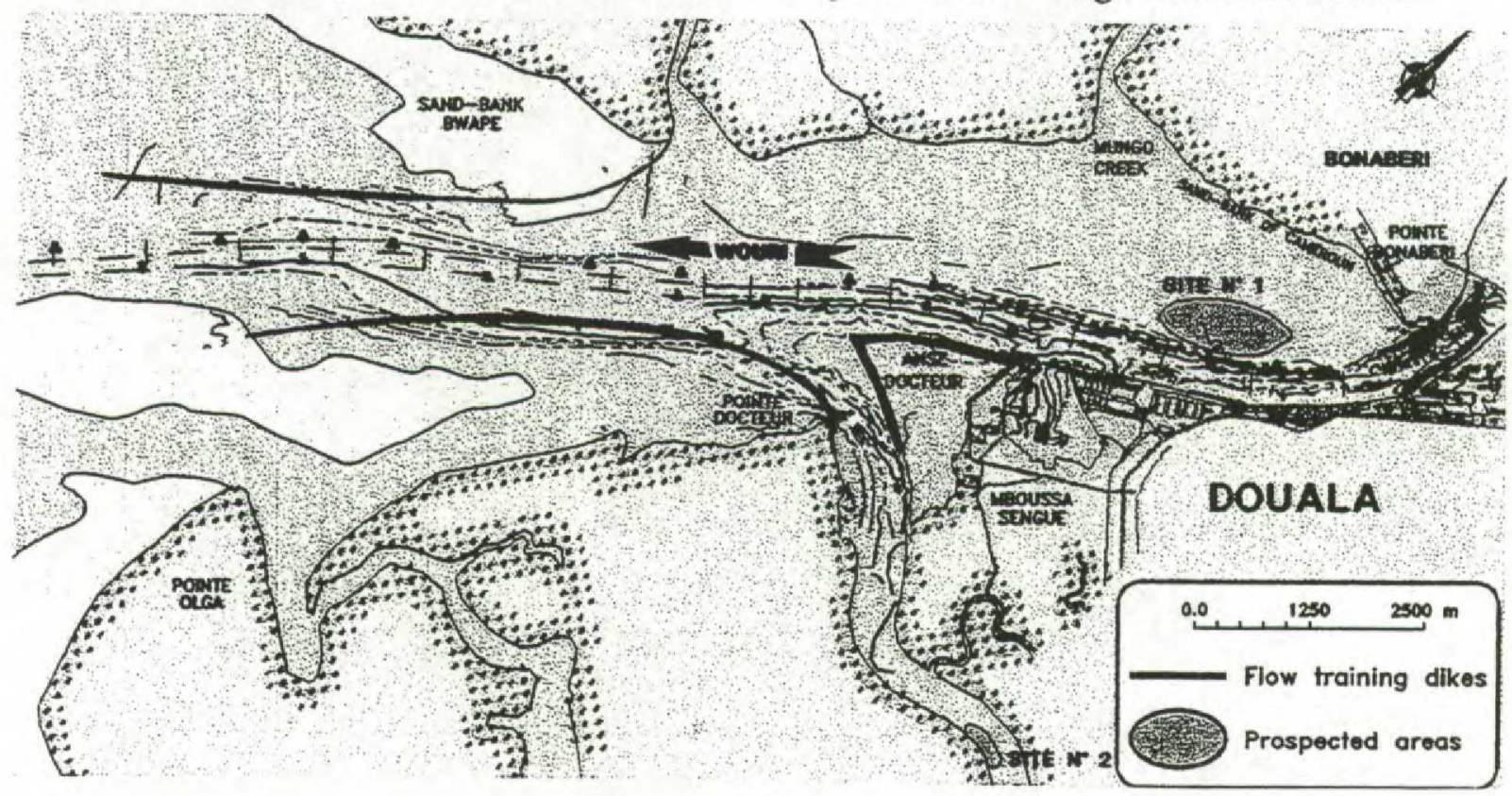




\section{PRINCIPLES OF THE METHOD}

DC methods are not very often used for marine prospection of first sediment layers. The two main reasons are firstly that traditionnal seismic reflexion methods are commonly used and seem to be well-adapted to this purpose and secondly that sea water is considered to be an electrical shunt making it impossible to perform DC measurements in sea sub-soil In fact, it is generally difficult, with seismic, to obtain good results in shallow water as well as in first sediment layers whereas DC prospection can provide information on first sediment layers if the contrast of resistivity between sea water and sediment is sufficient. It is generally the case as resistivity contrast varies typically from 1.5 to 10 , making it possible to consider DC propection as an alternative method for first sediment layers prospection.

For practical and theoritical reasons that we discuss hereafter, the measurement array is placed on the sea-bottom and not at the water surface. The array consists of 4 electrodes: A,B for current injection $I$ and $M, N$ for the measurement of potential differences $V m-V n$.

When the array is placed at the water surface, the apparent resistivity is $\rho_{a}=k \frac{V_{m}-V_{n}}{I}$

where $\mathbf{k}$ is the geometrical factor. When the array is placed on the water bottom, we shall take the same formula ( 1 ) for apparent resistivity, $k$ being the same as for surface array.

For a Wenner quadripole $\mathrm{AMNB}$ at the surface water, $\mathrm{k}=2 \pi \mathrm{a}(\mathrm{a}=\mathrm{AM}=\mathrm{MN}=\mathrm{NB})$ whereas it should be $k=4 \pi$ a when the array is placed on the water bottom because the current is injected at the interface between two media. In order to avoid more complexity, it is better to maintain the same value for $\mathrm{k}$, indeed $2 \pi \mathrm{a}$. Of course, we take this assumption into account in the theoritical model used for interpretation. This definition has the advantage of simplicity. It gives the same apparent resistivity values either the array is placed at the surface or on the bottom. If water depth tends towards zero (or what is equivalent if the array length tends towards infinity) or if water resistivity tends towards infinity, the apparent resitivities calculated with the two types of array tend towards the same limits.

In the case of a tabular medium measured with an array placed on water bottom, it has been shown in a previous paper (Lagabrielle and Teilhaud, 1981) how to calculate the apparent resistivity from the resolution of Laplace equation and how to calculate its limit conditions which are slightly different from those of a water surface array. It has been shown on the other hand (Lagabrielle, 1983) that a short water bottom array gives a better resolution in first sediment layers and that the two arrays (bottom and surface) are equivalent when long, whichever type of array is used: Wenner, Schlumberger, dipole-dipole.

In marine conditions, if we assume a two-layered medium with an insulating substratum $\left(\rho_{\infty}\right)$ topped by unconsolidated sediments $\left(\rho_{v}\right)$, much more conductive, the apparent resistivity is for a short array:

$$
\rho_{a}=\frac{\rho_{w} \rho_{v}}{\rho_{w}+\rho_{v}} \quad \text { (2) } \quad\left(\rho_{w} \text {, resistivity of water }\right)
$$

and becomes for a long array: (with d: water heigh, e: sediment thickness, $\mathrm{Ln}$ : Neperien log):

$$
\rho a=\frac{2 a \operatorname{Ln} 2}{\frac{d}{\rho w}+\frac{t}{\rho_{v}}}
$$


In logarithmic coordinates, it is the equation of a straight lime of slope 1 , intercepting the axis $\rho_{\mathrm{a}}=1$ for:

$$
a=\frac{1}{2 \operatorname{Ln} 2}\left[\frac{d}{\rho_{w}}+\frac{e}{\rho_{v}}\right]
$$

As it is shown in equation (3), the apparent resistivity for a long array is the same with electrodes located either on the sea-floor or at the water surface. If $d$ tends towards zero, equation (3) will be the same as if there is no water. Equation (2) shows that information on sediment resistivity is given by short array measurements whereas long array measurements do not provide such information.

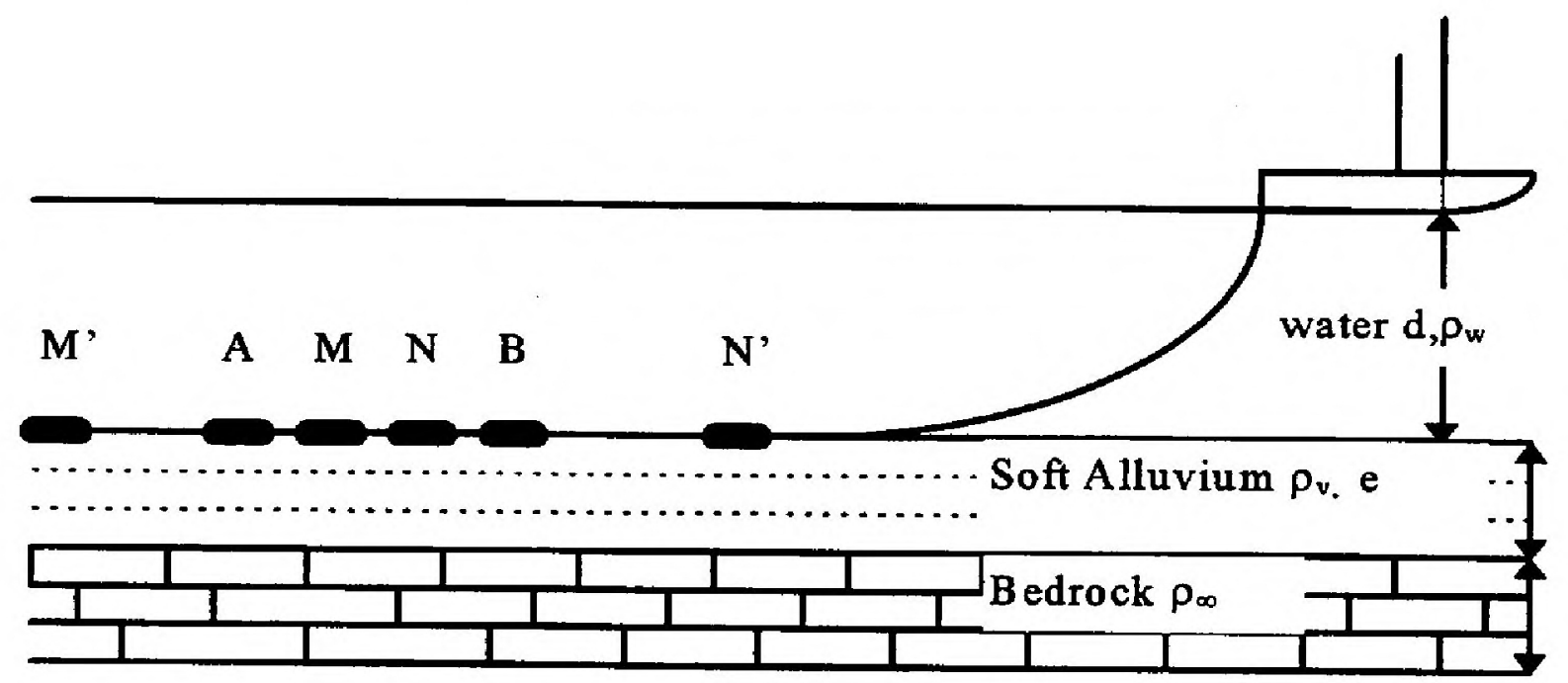

Figure 1: Electric array on sea bottom

In practice, $\mathrm{Vm}-\mathrm{Vn}$ and $\mathrm{Vm}$ '-Vn' are measured continuously along the profile and the apparent resistivities $\rho_{\mathrm{aMN}}, \rho_{\mathrm{aM}} \mathrm{N}^{\prime}$ are caculated using equation (1). For the interpretation, we determine, using equation (3) (d and $\rho_{w}$ are known) the values of longitudinal conductance $e / \rho_{v}$. The short Wenner array is used to verify the following hypothesis: tabularity of layers and invariability of water depth and resistivity; indeed, as the lengthes of the short and long Wenner arrays are in a ratio of three, apparent resistivities for the two arrays should be in the same ratio. If not, it means that layers are not tabular or that water depth or resistivity vary in an important way.

The variations of the conductance give information on the nature and thickness of the first layer. High conductance means important thickness or poor resistivity like in a silty context for instance. Low conductance means poor thickness or high resistivity as, for instance, in a sandy context. The results are calibrated using drillings to determine the actual nature and thickness of first layers.

In case of a tabular and homogeneous first sediment layer covering a substratum of infinite resistivity, it is possible to determine directly the thickness of the first layer if some core drillings are available. 


\section{APPLICATION IN THE WOURY ESTUARY}

We used a Wenner array with 2 electrodes, A and B, for I injection and 4 electrodes, M', M, $\mathrm{N}, \mathrm{N}^{\prime}$, for potential measurements. AMNB was a Wenner short array with $\mathrm{a}=23 \mathrm{~m}$. and

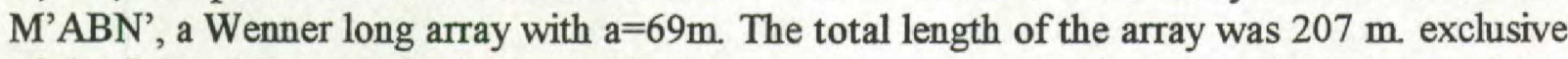
of the link cable towards the boat. The electrodes were made of copper pipes, $0.5 \mathrm{~m}$ long, moulded on the cable.

The cable was dragged on the sea bottom at a speed of 2 or 3 knots. The depth and resistivity of water were measured continuously with a sonar and a little Wenner probe placed under the boat. The X,Y position of the boat in the estuary was determined continuously using a Syledis positionning system. The tides were also measured with some scales placed all along the channel. A current generator located on board was producing the injection current I which was alternative $(0.1 \mathrm{~Hz}, 1$ to $5 \mathrm{~A})$ in order to face both the polarization of the electrodes and the spontaneous potentials phenomenas. The two differences of potential $\mathrm{Vm}-\mathrm{Vn}$ and $\mathrm{Vm}$ '-Vn' were recorded simultaneously on a paper plotter with high sensitivity and low frequency. In these conditions, 10 to $20 \mathrm{~km}$ of profile were measured every day.

The survey has been carried out during august 94 . For a total, $184 \mathrm{~km}$ of electrical profiles were recorded and 64 drillings with sediment samplings were performed to calibrate the geophysical data. Four areas were concerned for a total surface of $18 \mathrm{~km}^{2}$. One area showed no potentiality but the geophysical and geotechnical campaign has confirmed the presence of important quantities of sand and gravel on the three others areas with an estimation of 10 to 15 millions of $\mathrm{m}^{3}$. Moreover a complete bathymetric survey has been performed in order to determine the working conditions when dragging the estuary.

The geophysical and geotechnical survey ordered by CNPA has largely helped to confirm the faisability of the project, in term of sand and gravel material potentiality. The results of the survey are entire part of the tender for construction of the flow training dikes.

\section{LONGITUDINAL CONDUCTANCE - ZONE 1}

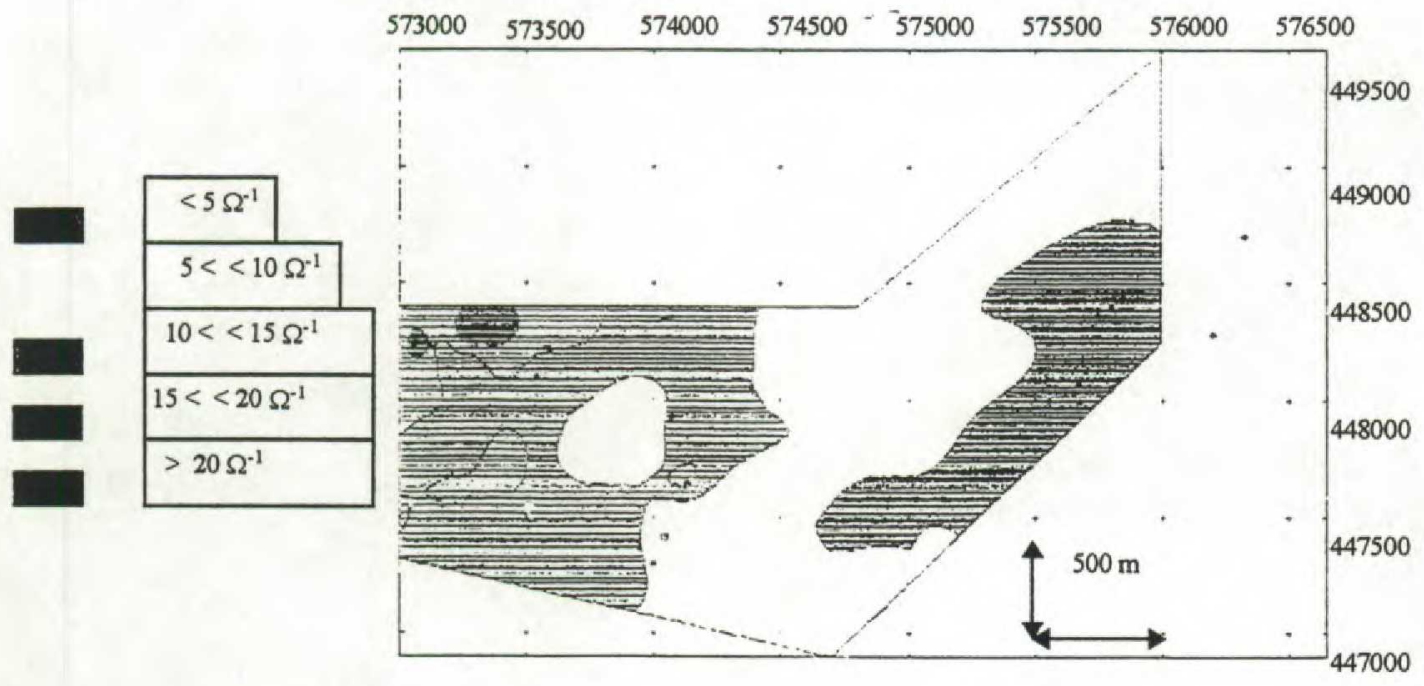

